\title{
Widdrol induces apoptosis via activation of AMP-activated protein kinase in colon cancer cells
}

\author{
MOO RIM KANG ${ }^{1 *}$, SONG-KYU PARK ${ }^{2 *}$, CHANG WOO LEE ${ }^{1}$, IG JUN CHO ${ }^{1}$, YEONG NANG JO ${ }^{1}$, \\ JEONG WOOK YANG ${ }^{1}$, JIN-AH KIM ${ }^{1}$, JIEUN YUN ${ }^{1}$, KI HOON LEE ${ }^{1}$, HYUN JU KWON $^{3}$, \\ BYUNG WOO KIM ${ }^{3}$, KIHO LEE ${ }^{2}$, JONG SOON KANG ${ }^{1}$ and HWAN MOOK KIM ${ }^{4}$ \\ ${ }^{1}$ Bioevaluation Center, Korea Research Institute of Bioscience and Biotechnology, Ochang, Cheongwon, \\ Chungbuk 363-883; ${ }^{2}$ Department of Pharmacy, College of Pharmacy, Korea University, Jochiwon, Yeongi, \\ Chungnam 339-700; ${ }^{3}$ Department of Life Science and Biotechnology, College of Natural Science, Dong-Eui \\ University, Eomgwang, Busan-Jin, Busan 614-714; ${ }^{4}$ Department of Pharmacy, College of Pharmacy, \\ Gachon University of Medicine and Science, Yeonsu, Incheon 406-799, Republic of Korea
}

Received November 24, 2011; Accepted December 29, 2011

DOI: 10.3892/or.2012.1644

\begin{abstract}
Widdrol, a natural sesquiterpene present in Juniperus sp., has been shown to exert anticancer and antifungal effects. Emerging evidence has suggested that AMP-activated protein kinase (AMPK), which functions as a cellular energy sensor, is a potential therapeutic target for human cancers. In this study, we found that AMPK mediates the anticancer effects of widdrol through induction of apoptosis in HT-29 colon cancer cells. We showed that widdrol induced the phosphorylation of AMPK in a dose- and time-dependent manner. The selective AMPK inhibitor compound $\mathrm{C}$ abrogated the inhibitory effect of widdrol on HT-29 cell growth. In addition, we demonstrated that widdrol induced apoptosis and this was associated with the activation of caspases, including caspase-3/7 and caspase-9, in HT-29 cells. We also demonstrated that transfection of HT-29 cells with AMPK siRNAs significantly suppressed the widdrol-mediated apoptosis and the activation of caspases. However, cell cycle arrest induced by widdrol was not affected by transfection of HT-29 cells with AMPK siRNAs. Furthermore, widdrol inhibited HT-29 tumor growth in a human tumor xenograft model. Taken together,
\end{abstract}

Correspondence to: Dr Jong Soon Kang, Bioevaluation Center, Korea Research Institute of Bioscience and Biotechnology, Ochang, Cheongwon, Chungbuk 363-883, Republic of Korea

E-mail: kanjon@kribb.re.kr

Dr Hwan Mook Kim, Department of Pharmacy, College of Pharmacy, Gachon University of Medicine and Science, Yeonsu,Incheon 406-799, Republic of Korea

E-mail: hwanmook@gachon.ac.kr

${ }^{*}$ Contributed equally

Key words: widdrol, AMP-activated protein kinase, colon cancer, apoptosis our results suggest that the anticancer effect of widdrol may be mediated, at least in part, by induction of apoptosis via AMPK activation.

\section{Introduction}

AMP-activated protein kinase (AMPK), a serine/threonine protein kinase conserved in eukaryotes, is known as a cellular energy sensor involved in the regulation of the response to environmental or nutritional stress (1). AMPK is a heterotrimeric complex composed of catalytic $\alpha$ subunit and regulatory $\beta$ and $\gamma$ subunits. AMPK is activated in response to energy deprivation and the activation of AMPK regulates a variety of cellular processes, such as hepatic fatty acid oxidation, lipogenesis, glycogen synthesis and glucose uptake (2). Recently, AMPK has also been identified as a potential target for cancer prevention and/or treatment (3). Indeed, a variety of AMPK activators have been shown to exert anticancer effects (4-6). Especially, metformin, an orally available biguanide derivative that is widely used for the treatment of Type 2 diabetes, was shown to exert anticancer effects by activation of AMPK and is currently being investigated in clinical trials (4). AMPK is also reported to be linked with tumor suppressors, such as liver kinase B1 (LBK1), p21, p53 and tuberous sclerosis complex 2 (TSC2), indicating that AMPK is a potential therapeutic target for cancer (7-9).

The leaves of Juniperus chinensis have been reported to contain a lignan with strong anti-leukemic and antitumor activities (10) and a sesquiterpene with anti-fungal activity (11). Widdrol is an odorant derivative contained in various plants of Juniperus sp., including J. chinensis and J. lucayana $(12,13)$. In a previous study, widdrol was shown to induce cell cycle arrest in HT-29 cells (13). To find additional mechanisms involved in the anticancer activity of widdrol, we examined whether widdrol induces apoptosis and characterized molecular mechanism responsible for this effect. We also examined the effect of widdrol on tumor growth in a human tumor xenograft model. 


\section{Materials and methods}

Chemicals. All reagents were purchased from Sigma-Aldrich (St. Louis, MO, USA) unless otherwise stated. Widdrol used in this study was isolated from J. chinensis as previously described (Fig. 1) (13). Widdrol was dissolved in dimethyl sulfoxide (DMSO) and freshly diluted in culture media for all in vitro experiments. For in vivo experiments, widdrol was dissolved in a solution containing $10 \%$ ethanol and $10 \%$ Cremophor EL.

Cell culture and transient transfection. The human colon adenocarcinoma cell line HT-29 was purchased from American Type Culture Collection (ATCC, Rockville, MD) and was cultured in RPMI-1640 medium (Gibco-BRL, Grand Island, NY, USA) supplemented with $10 \%$ fetal bovine serum (Hyclone, Logan, UT, USA), $2 \mathrm{mM} \mathrm{L-glutamine} \mathrm{and} 50 \mu \mathrm{g} / \mathrm{ml}$ of gentamycin (Gibco-BRL). Cells were maintained at $37^{\circ} \mathrm{C}$ in $5 \% \mathrm{CO}_{2}$ humidified air. AMPK siRNA was purchased from Bioneer (Daejon, Korea) and cells were transfected using Lipofectamine RNAiMAX (Invitrogen Life Technologies, Carlsbad, CA, USA) according to the manufacturer's instructions.

Cell proliferation assay. Cells were plated at $8 \times 10^{3}$ cells/well in 96-well plates, incubated overnight, and treated with widdrol for $48 \mathrm{~h}$. Cell proliferation assays were performed using a Cell Proliferation Kit II (Roche Applied Science, Mannheim, Germany) according to the manufacturer's instructions. Briefly, the XTT labeling mixture was prepared by mixing 50 volumes of $1 \mu \mathrm{g} / \mathrm{ml}$ sodium 3'-[1-(phenylaminocarbonyl)-3,4-tetrazolium]bis (4-methoxy-6-nitro) benzene sulfonic acid hydrate with 1 volume of $0.383 \mu \mathrm{g} / \mathrm{ml}$ of $\mathrm{N}$-methyldibenzopyrazine methyl sulfate. This XTT labeling mixture was added to the cultures and incubated for $2 \mathrm{~h}$ at $37^{\circ} \mathrm{C}$. Absorbance was measured at $490 \mathrm{~nm}$ with a reference wavelength at $650 \mathrm{~nm}$.

Apoptosis analysis. Apoptosis analysis was performed using an Annexin V-FITC Apoptosis Detection Kit II (BD Bioscience, San Jose, CA, USA) according to the manufacturer's instructions. Briefly, cells were plated at $2 \times 10^{5}$ cells/ well in 6-well plates, incubated overnight, and treated with the indicated concentrations of widdrol for $24 \mathrm{~h}$. Cells were harvested, washed with PBS, and combined with a binding buffer containing Annexin-V-FITC and propidium iodide. Following a 15-min incubation in the dark, cells were analyzed by flow cytometry using a FACSCalibur flow cytometer (BD Bioscience).

Caspase activity assay. The activities of caspases were determined using a Caspase-Glo ${ }^{\mathrm{TM}}$ Assay (Promega, Madison, WI, USA) according to the manufacturer's instructions. Briefly, cells were plated at $8 \times 10^{3}$ cells/well in 96-well plates, incubated overnight and treated with the indicated concentrations of widdrol for $24 \mathrm{~h}$. The culture supernatants were transferred to 96 -well turbid microtiter plates and $50 \mu \mathrm{l}$ of proluminescent caspase-3/7 and caspase- 9 substrate were added. After $1 \mathrm{~h}$ of incubation at $37^{\circ} \mathrm{C}$, the luminescence was measured using a VICTOR $^{\text {TM }}$ Light system (Perkin-Elmer, Waltham, MA, USA).

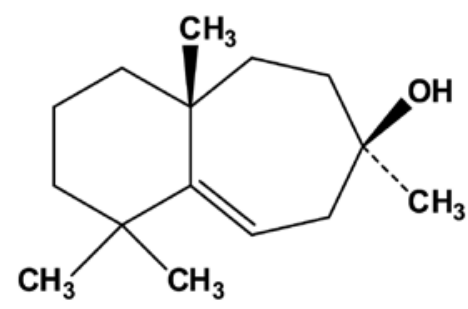

Figure 1. Chemical structure of widdrol.

Western immunoblot analysis. Total protein extracts were prepared by lysing cells in lysis buffer $(50 \mathrm{mM}$ Tris- $\mathrm{HCl}$ (pH 7.4), $150 \mathrm{mM} \mathrm{NaCl}, 1 \mathrm{mM}$ EGTA, 5 mM EDTA, 0.5\% Triton X-100, $0.25 \%$ sodium deoxycholate, $1 \mathrm{mM}$ sodium fluoride, $1 \mathrm{mM}$ sodium orthovanadate, $5 \mu \mathrm{g} / \mathrm{ml}$ leupeptin, $0.2 \mathrm{mM}$ phenylmethylsulfonyl fluoride, $10 \mu \mathrm{g} / \mathrm{ml}$ aprotinin, $0.5 \mu \mathrm{M}$ DTT) and protein concentrations in the lysates were determined using a Bio-Rad protein assay kit (Bio-Rad Laboratories, Inc., Hercules, CA, USA) according to the manufacturer's instructions. Protein extracts were resolved by $12 \%$ SDS-polyacrylamide gel electrophoresis and transferred to nitrocellulose membranes. The membranes were incubated with blocking buffer (Tris-buffered saline containing $0.2 \%$ Tween-20 and 3\% non-fat dried milk) and probed with the indicated primary antibodies. After washing, membranes were probed with horseradish peroxidase-conjugated secondary antibodies. Detection was performed using an enhanced chemiluminescent protein (ECL) detection system (Amersham Biosciences, Little Chalfont, UK).

Cell cycle analysis. Cell cycle analysis was performed using a previously described protocol (14). Briefly, cells were plated at $3 \times 10^{6}$ cells/dish in $100-\mathrm{mm}$ dishes, incubated overnight, and treated with the indicated concentrations of widdrol. After $24 \mathrm{~h}$, cells were harvested and washed with PBS. After cell counting with trypan blue staining, $1 \times 10^{6}$ cells were pelleted and fixed in $70 \%$ ethanol at $4^{\circ} \mathrm{C}$ for $1 \mathrm{~h}$. Then cells were resuspended $1 \mathrm{ml}$ of Krishan's buffer $(0.1 \%$ sodium citrate, $0.02 \mathrm{mg} / \mathrm{ml}$ RNase A, $0.3 \%$ Triton X-100, and $50 \mu \mathrm{g} / \mathrm{ml}$ propidium iodide, $\mathrm{pH}$ 7.4) for $1 \mathrm{~h}$ at $4^{\circ} \mathrm{C}$. Samples were centrifuged, resuspended in $1 \mathrm{ml}$ of PBS buffer, and analyzed by flow cytometry using a FACSCalibur flow cytometer (Becton-Dickinson, San Jose, CA, USA). Data were collected for 10,000 events. The Modfit LT program (Verity Software House, Maine, ME, USA) was used for cell cycle modeling.

Human tumor xenograft model. Specific pathogen-free BALB/ c-nu female mice (SLC, Hamamatsu, Japan), 5-weeks old and weighing from 15.5 to $16.5 \mathrm{~g}$, were maintained as previously described (15). All animals were allowed to acclimatize to the local environment for at least 1 week before use. The mice were randomly assigned to three groups, all of which were subcutaneously inoculated with $0.3 \mathrm{ml}$ of HT-29 cells $\left(3 \times 10^{7}\right.$ cells $\left./ \mathrm{ml}\right)$ on Day 0 . Widdrol $(50 \mathrm{mg} / \mathrm{kg})$ was administered i.p. daily and doxorubicin $(2 \mathrm{mg} / \mathrm{kg})$ was administered i.p. on alternate days for 20 days. The volume of tumors was measured every other day with a vernier caliper and then calculated according to the formula: tumor volume (in $\mathrm{mm}^{3}$ ) $=$ [length (in $\mathrm{mm}$ ) $\mathrm{x}$ width (in 
A

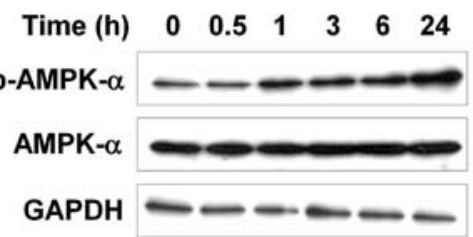

B
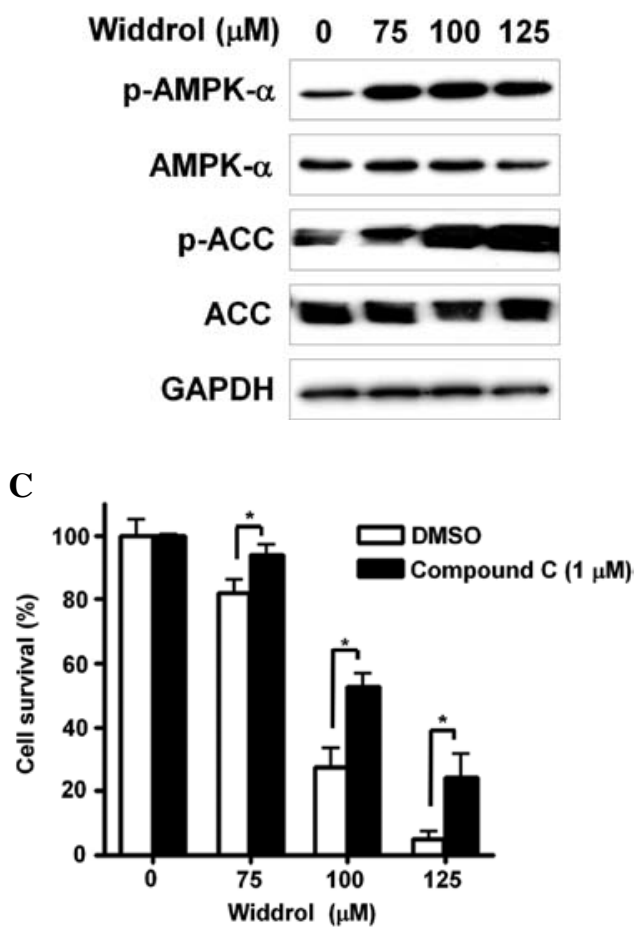

Figure 2. Effects of widdrol on AMPK activation. HT-29 cells were treated with $100 \mu \mathrm{M}$ of widdrol for the indicated times (A) or with the indicated concentrations of widdrol for $24 \mathrm{~h}$ (B). Cell lysates were subjected to immunoblotting using antibodies specific to phosphorylated AMPK- $\alpha$ (Thr172), AMPK- $\alpha$, phosphorylated ACC (Ser79), ACC and GAPDH. (C) Cells were pretreated with vehicle or compound $\mathrm{C}(1 \mu \mathrm{M})$ and then treated with the indicated concentrations of widdrol for $24 \mathrm{~h}$. Cell proliferation was determined by XTT assay. Results are presented as the mean \pm SD of triplicate determinations $($ ( $\mathrm{P}<0.05)$.

$\mathrm{mm}$ ) $\mathrm{x}$ height (in $\mathrm{mm}$ )]/2. All mice were weighed every other day. After the treatment for 20 days, the mice were euthanized, and all tumors were removed and weighed.

Statistical analysis. Results are expressed as the mean \pm SD. A paired t-test was used to compare two groups, and one-way ANOVA followed by Dunnett's t-test was used for multiple comparisons using GraphPad Prism (GraphPad Software, Inc., San Diego, CA, USA). The criterion for statistical significance was set at $\mathrm{P}<0.05$.

\section{Results}

Induction of AMPK phosphorylation by widdrol in HT-29 cells. Recently, several reports demonstrated that the activation of AMPK is related to cancer cell death in various cancer cell lines $(16,17)$. Therefore, we investigated whether the antitumor activity of widdrol is accompanied by AMPK activation. HT-29 cells were treated with $100 \mu \mathrm{M}$ of widdrol for up
A

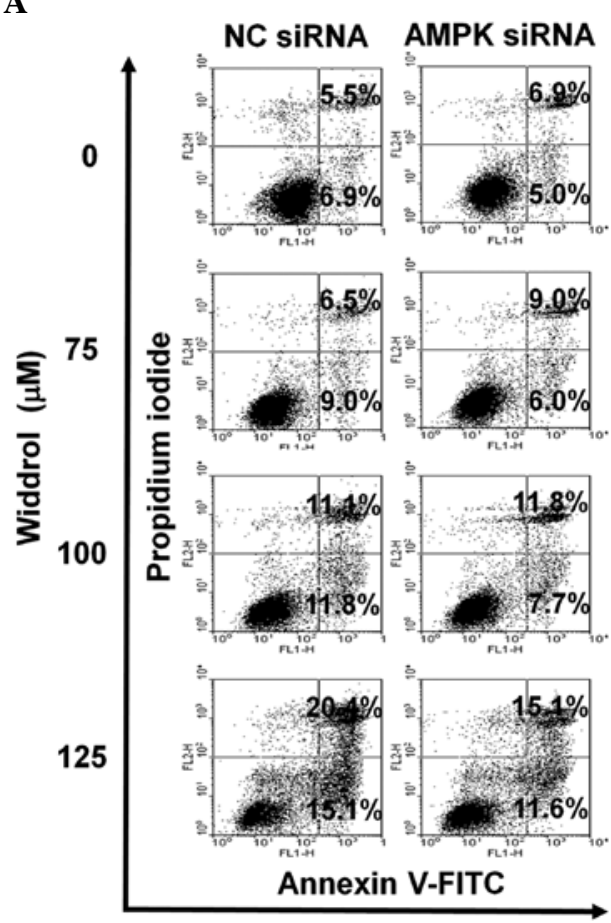

B

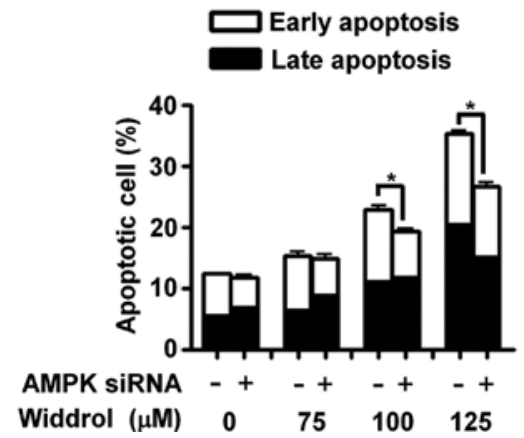

Figure 3. Induction of apoptosis by widdrol. HT-29 cells were transfected with AMPK siRNA or negative control (NC) siRNA and then treated with the indicated concentrations of widdrol for $24 \mathrm{~h}$. (A) Cells were stained with propidium iodide and FITC-conjugated antibody directed against Annexin-V and analyzed by flow cytometry. (B) The flow cytometry results were analyzed and expressed as bar graphs. Results are presented as the mean \pm SD of triplicate determinations $(" \mathrm{P}<0.05)$.

to $24 \mathrm{~h}$ or were exposed with the indicated concentrations of widdrol for $24 \mathrm{~h}$ to examine the time- and dose-related effects of widdrol on AMPK phosphorylation. As shown in Fig. 2A and $\mathrm{B}$, widdrol markedly stimulated the phosphorylation of AMPK- $\alpha$ in a time- and dose-dependent manner in HT-29 cells. Fig. 2B also shows that the phosphorylation of acetylCoA carboxylase (ACC), one of the main downstream factors of AMPK, was also induced by widdrol in a dose-dependent manner. Widdrol treatment did not affect the expression of AMPK- $\alpha$, ACC and GAPDH in HT-29 cells (Fig. 2B). To further investigate whether widdrol inhibits cancer cell growth by AMPK activation, we examined the effect of compound $\mathrm{C}$, a well-known AMPK inhibitor, on widdrol-mediated inhibition of cell growth in HT-29 cells. As shown in Fig. 2C, compound C significantly reversed widdrol-mediated inhibition of cell growth in HT-29 cells. 
A

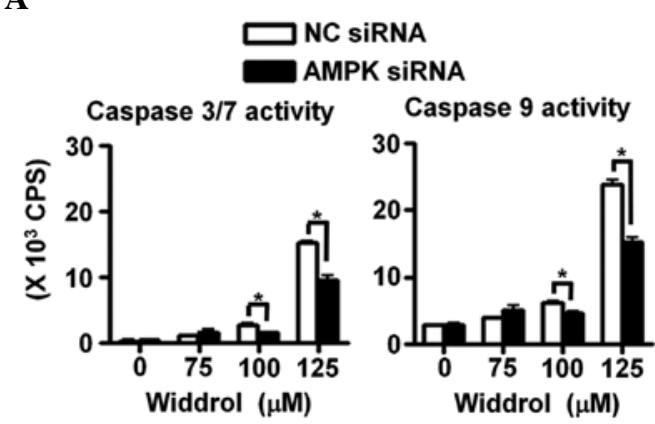

B

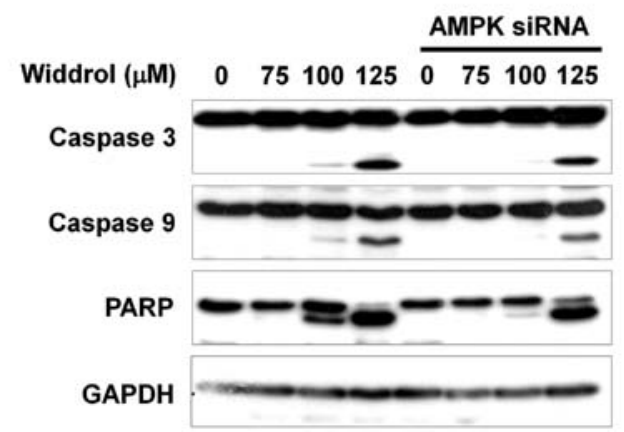

Figure 4. Activation of caspases by widdrol. HT-29 cells were transfected with AMPK siRNA or negative control (NC) siRNA and then treated with the indicated concentrations of widdrol for $24 \mathrm{~h}$. (A) Culture supernatants were collected, and the activities of caspase-3/7 and caspase- 9 were determined. Results are presented as the mean \pm SD of triplicate determinations ( $\mathrm{P}<0.05)$. (B) Levels of caspase-3, caspase-9, PARP and GAPDH in the total cell lysates were determined via western immunoblot analysis.

AMPK-dependent induction of apoptosis by widdrol in HT-29 cells. In this study, we investigated whether the induction of apoptosis is involved in widdrol-mediated inhibition of cancer cell growth. As shown in Fig. 3, widdrol induced apoptosis in a concentration-dependent manner; 9.0, 11.8 and $15.1 \%$ of the HT-29 cells were Annexin-V-positive and propidium iodidenegative after exposure at 75,100 and $125 \mu \mathrm{M}$ of widdrol, respectively. To investigate whether the activation of AMPK is involved in widdrol-induced apoptosis, the cells were transfected with AMPK siRNA and treated with widdrol for $24 \mathrm{~h}$. Fig. 3 shows that apoptosis of HT-29 cells by widdrol was significantly reduced by knockdown of AMPK expression.

AMPK-dependent activation of caspases by widdrol in HT-29 cells. We also assessed whether widdrol activates caspases, a key enzyme involved in apoptotic signaling cascade. As shown in Fig. 4A, widdrol dose-dependently induced the activation of caspase-3/7 and caspase-9. However, transfection of cells with AMPK siRNA reversed the activation of caspases by widdrol in HT-29 cells. We also confirmed that widdrol induces the cleavage of caspase-3, caspase- 9 and poly(ADP-ribose) polymerase (PARP) by western immunoblot analysis in HT-29 cells (Fig. 4B).

AMPK-independent induction of cell cycle arrest by widdrol in HT-29 cells. It has been reported that widdrol inhibits cancer cell growth through the induction of G0/G1 phase
A

\section{NC SIRNA AMPK siRNA}

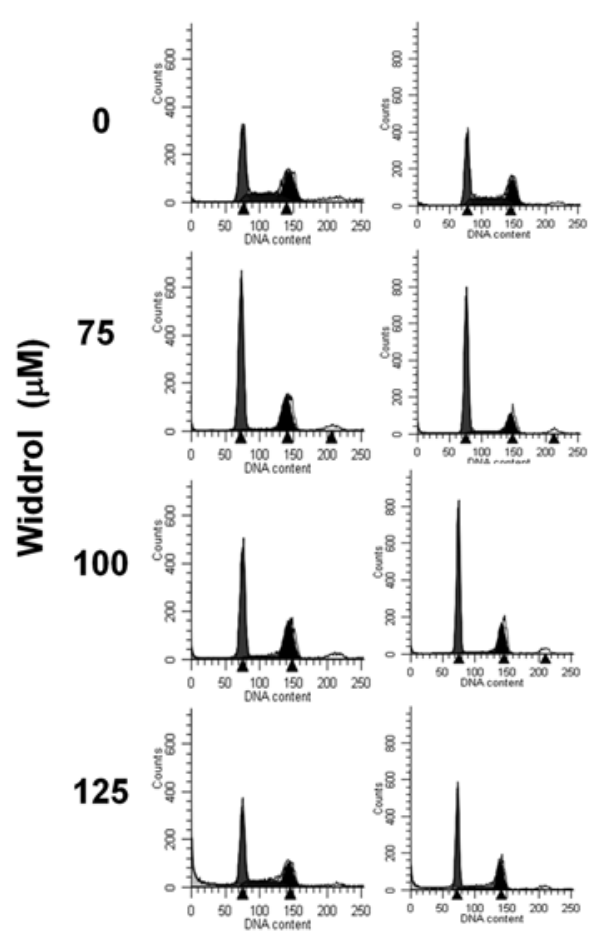

B

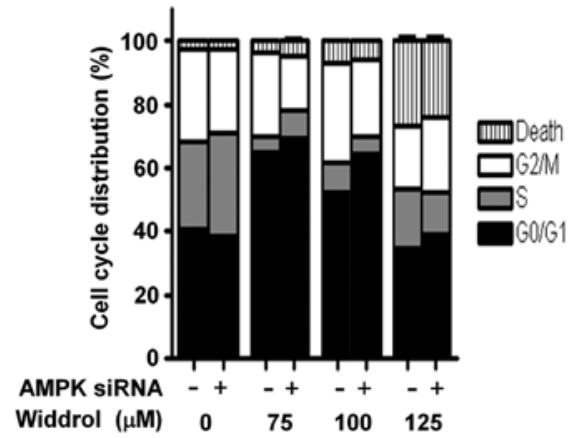

Figure 5. Induction of cell cycle arrest by widdrol. HT-29 cells were transfected with AMPK siRNA or negative control (NC) siRNA and then treated with the indicated concentrations of widdrol for $24 \mathrm{~h}$. Cell cycle distribution was examined by flow cytometry and data were analyzed using Modfit LT. (A) A representative result is shown. (B) Results are presented as the mean $\pm \mathrm{SD}$ of triplicate determinations $\left({ }^{*} \mathrm{P}<0.05\right)$

cell cycle arrest (13). In a further investigation, we examined whether widdrol-induced cell cycle arrest is mediated by AMPK. As shown in Fig. 5, widdrol induced G0/G1 phase cell cycle arrest at low doses and cell death at high doses in HT-29 cells. However, transfection of HT-29 cells with AMPK siRNAs does not significantly affect widdrol-induced cell cycle arrest.

Inhibition of tumor growth by widdrol in a human tumor xenograft model. To determine if widdrol would exert an antitumor effect in vivo, the effect of widdrol on HT-29 tumor growth was evaluated in human tumor xenograft model. Widdrol and doxorubicin inhibited HT-29 tumor growth by 42.3 and $38.1 \%$, respectively, compared to vehicle-treated group (Fig. 6A). Fig. 6C shows that tumor weight was also 
A

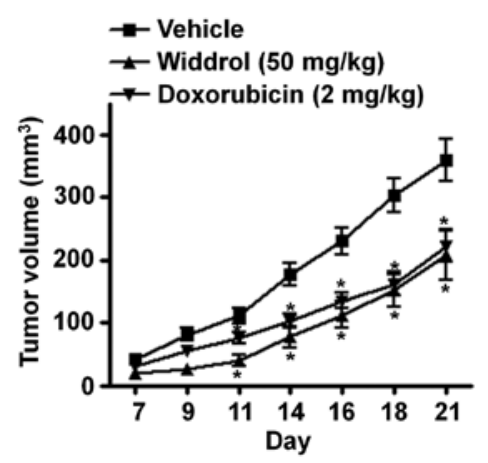

B

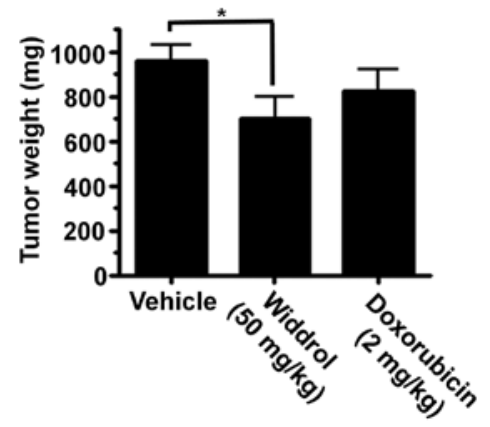

C

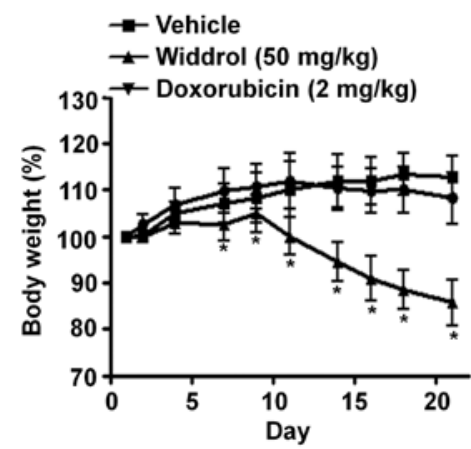

Figure 6. Inhibition of the HT-29 tumor growth by widdrol in a human tumor xenograft model. Specific pathogen-free BALB/c-nu female mice $(n=10)$ were implanted subcutaneously with nine million HT-29 colon cancer cells. From the next day, $50 \mathrm{mg} / \mathrm{kg}$ of widdrol and $2 \mathrm{mg} / \mathrm{kg}$ of doxorubicin (DOX) were injected intraperitoneally every day and every other day, respectively. (A) Tumor volumes were measured three times a week and estimated as described in Materials and methods. (B) Tumor weights were measured and expressed as bar graphs. (C) The body weights of the tumor bearing nude mice were measured three times a week. Results are presented as the mean \pm SD of 10 determinations $\left({ }^{*} \mathrm{P}<0.05\right)$.

significantly reduced by widdrol treatment. The body weights of tumor-bearing nude mice were examined to assess the toxicity of widdrol. No significant difference in body weights between vehicle-treated group and widdrol-treated group was observed during the period of the experiment, whereas the body weights of the doxorubicin-treated group was decreased to $85.7 \%$ of the initial body weights (Fig. 6B).

\section{Discussion}

Caloric restriction reduces the incidence and progression of spontaneous and induced tumors in laboratory rodents. One of the key enzymes thought to be involved in this process is
AMPK, a regulator of cellular energy homeostasis. AMPK is activated under conditions of elevated AMP/ATP ratio, such as hypoxia, ischemia and glucose deprivation $(1,18)$. Recently, it has been reported that mimicking a low energy status by treatment with a cell-permeable nucleoside 5-aminoimidazone-4-carboxamide riboside inhibited cell proliferation and suppressed malignant phenotype in cancer cells (19). Huang et al also reported that pharmacological activation of AMPK resulted in a decrease in endogenous lipogenesis, an inhibition of cell proliferation and a suppression of tumorigenic and invasive properties in cancer cells in vitro and in vivo (20), suggesting that AMPK might be a potential therapeutic target for the treatment of cancer. In the present study, we clearly demonstrated that widdrol induces the activation of AMPK and subsequent phosphorylation of ACC in HT-29 cells. In addition, a blockade of AMPK activation by treatment of HT-29 cells with compound $\mathrm{C}$, a well-known inhibitor of AMPK, reversed widdrol-mediated cancer cell death, suggesting that the anticancer effect of widdrol might be linked with its ability to induce AMPK.

It has been revealed that AMPK is involved in apoptosis (21). Kim et al reported that the activation of AMPK by compound $\mathrm{K}$ induced apoptosis and compound K-mediated apoptosis was blunted in the presence of either an inhibitor of AMPK or a small interfering RNA for AMPK (22). In addition, Khanal et al reported p-HPEA-EDA, a phenolic compound of virgin olive oil, reduced cell viability and induced apoptosis by upregulation of PARP and caspase- 3 in colon cancer cells through activation of AMPK (23). These results suggest that pharmacological modulation of AMPK might induce apoptosis in cancer cells. In the present study, we demonstrated that widdrol induced apoptosis in HT-29 cells. Widdrol also induced the activity of caspases, including caspase-3/7 and caspase-9. However, widdrol-induced apoptosis and caspase activation in HT-29 cells were suppressed by transfection of a small interfering RNA for AMPK, suggesting that widdrolinduced apoptosis is mediated, at least in part, by AMPK activation.

Previous report demonstrated that widdrol inhibits HT-29 cell proliferation via inducing cell cycle arrest (13). In this report, they showed that the induction of cell cycle arrest by widdrol was correlated with the induction of p53 phosphorylation and p21 expression. Here, we also investigated the relationship between widdrol-induced cell cycle arrest and AMPK activation. Consistent with previous reports, we showed that widdrol induces G0/G1 phase cell cycle arrest in HT-29 cells. However, our results demonstrated that the transfection of a small interfering RNA for AMPK does not affect widdrol-induced cell cycle arrest in HT-29 cells, suggesting that cell cycle arrest by widdrol is independent of widdrolmediated induction of AMPK.

In conclusion, the present study demonstrates that widdrol inhibited the growth of HT-29 colon cancer cells by inducing apoptosis and this is mediated, at least in part, by activation of AMPK by widdrol. In addition, we also showed that widdrol could inhibit the growth of HT-29 tumors in a human tumor xenograft model. These findings provide a molecular mechanism involved in the anticancer effect of widdrol and suggest that widdrol is a potential chemotherapeutic candidate for cancer treatment. 


\section{Acknowledgements}

This study was supported in part by grants (no. A101836) from the Korea Health Technology R\&D Project, Ministry of Health, Welfare and Family Affairs, Republic of Korea and the KRIBB Research Initiative Program.

\section{References}

1. Koh HJ, Brandauer J and Goodyear LJ: LKB1 and AMPK and the regulation of skeletal muscle metabolism. Curr Opin Clin Nutr Metab Care 11: 227-232, 2008.

2. Shaw RJ, Kosmatka M, Bardeesy N, et al: The tumor suppressor LKB1 kinase directly activates AMP-activated kinase and regulates apoptosis in response to energy stress. Proc Natl Acad Sci USA 101: 3329-3335, 2004.

3. Fogarty S and Hardie DG: Development of protein kinase activators: AMPK as a target in metabolic disorders and cancer. Biochim Biophys Acta 1804: 581-591, 2010.

4. Guppy A, Jamal-Hanjani M and Pickering L: Anticancer effects of metformin and its potential use as a therapeutic agent for breast cancer. Future Oncol 7: 727-736, 2011.

5. Kim AD, Kang KA, Zhang R, et al: Ginseng saponin metabolite induces apoptosis in MCF-7 breast cancer cells through the modulation of AMP-activated protein kinase. Environ Toxicol Pharmacol 30: 134-140, 2010.

6. Rocha GZ, Dias MM, Ropelle ER, et al: Metformin amplifies chemotherapy-induced AMPK activation and antitumoral growth. Clin Cancer Res 17: 3993-4005, 2011.

7. Rattan R, Giri S, Singh AK and Singh I: 5-Aminoimidazole4-carboxamide-1-beta-D-ribofuranoside inhibits cancer cell proliferation in vitro and in vivo via AMP-activated protein kinase. J Biol Chem 280: 39582-39593, 2005.

8. Luo Z, Saha AK, Xiang X and Ruderman NB: AMPK, the metabolic syndrome and cancer. Trends Pharmacol Sci 26: 69-76, 2005.

9. Su RY, Chao Y, Chen TY, Huang DY and Lin WW: 5-Aminoimidazole-4-carboxamide riboside sensitizes TRAILand TNF- $\alpha$-induced cytotoxicity in colon cancer cells through AMP-activated protein kinase signaling. Mol Cancer Ther 6: 1562-1571, 2007.

10. Ali AM, Mackeen MM, Intan-Safinar I, et al: Antitumourpromoting and antitumour activities of the crude extract from the leaves of Juniperus chinensis. J Ethnopharmacol 53: 165-169, 1996.
11. Nunez YO, Salabarria IS, Collado IG and Hernandez-Galan R: The antifungal activity of widdrol and its biotransformation by Colletotrichum gloeosporioides (penz.) Penz. \& Sacc. and Botrytis cinerea Pers.: Fr. J Agric Food Chem 54: 7517-7521, 2006.

12. Nunez YO, Salabarria IS, Collado IG and Hernandez-Galan R: Sesquiterpenes from the wood of Juniperus lucayana. Phytochemistry 68: 2409-2414, 2007.

13. Kwon HJ, Hong YK, Park C, et al: Widdrol induces cell cycle arrest, associated with MCM down-regulation, in human colon adenocarcinoma cells. Cancer Lett 290: 96-103, 2010.

14. Krishan A: Rapid flow cytofluorometric analysis of mammalian cell cycle by propidium iodide staining. J Cell Biol 66: 188-193, 1975.

15. Kim HM, Lim J, Yoon YD, et al: Antitumor activity of ex vivo expanded cytokine-induced killer cells against human hepatocellular carcinoma. Int Immunopharmacol 7: 1793-1801, 2007.

16. Sai K, Yang D, Yamamoto $\mathrm{H}$, et al: $\mathrm{A} 1$ adenosine receptor signal and AMPK involving caspase-9/-3 activation are responsible for adenosine-induced RCR-1 astrocytoma cell death. Neurotoxicology 27: 458-467, 2006.

17. Hwang JT, Ha J, Park IJ, et al: Apoptotic effect of EGCG in HT-29 colon cancer cells via AMPK signal pathway. Cancer Lett 247: 115-121, 2007.

18. Hardie DG: The AMP-activated protein kinase pathway - new players upstream and downstream. J Cell Sci 117: 5479-5487, 2004.

19. Swinnen JV, Beckers A, Brusselmans K, et al: Mimicry of a cellular low energy status blocks tumor cell anabolism and suppresses the malignant phenotype. Cancer Res 65: 2441-2448, 2005.

20. Huang CH, Tsai SJ, Wang YJ, Pan MH, Kao JY and Way TD: EGCG inhibits protein synthesis, lipogenesis, and cell cycle progression through activation of AMPK in p53 positive and negative human hepatoma cells. Mol Nutr Food Res 53: 1156-1165, 2009.

21. Hardie DG: New roles for the LKB1 $\rightarrow$ AMPK pathway. Curr Opin Cell Biol 17: 167-173, 2005.

22. Kim do Y, Park MW, Yuan HD, Lee HJ, Kim SH and Chung SH: Compound $\mathrm{K}$ induces apoptosis via CAMK-IV/AMPK pathways in HT-29 colon cancer cells. J Agric Food Chem 57: 10573-10578, 2009.

23. Khanal P, Oh WK, Yun HJ, et al: p-HPEA-EDA, a phenolic compound of virgin olive oil, activates AMP-activated protein kinase to inhibit carcinogenesis. Carcinogenesis 32: 545-553, 2011. 